\title{
Radiometer monitoring system for partial discharge detection in substation.
}

\author{
ZHANG, Y., NETO, J.M., UPTON, D., JABER, A., KHAN, U., SAEED, B., \\ AHMED, H., MATHER, P., ATKINSON, R., NETO, J.S., VIEIRA, M.F.Q., \\ LAZARIDIS, P. and GLOVER, I.A.
}

(C) 2015 IEEE. Personal use of this material is permitted. Permission from IEEE must be obtained for all other uses, in any current or future media, including reprinting/republishing this material for advertising or promotional purposes, creating new collective works, for resale or redistribution to servers or lists, or reuse of any copyrighted component of this work in other works. 


\title{
Radiometer Monitoring System for Partial Discharge Detection in Substation
}

\author{
Y. Zhang ${ }^{1}$, J.M. Neto ${ }^{3}$, D. Upton ${ }^{1}$, A. Jaber ${ }^{1}$, U. Khan ${ }^{1}$, B. Saeed ${ }^{1}$, H. Ahmed ${ }^{1}$, \\ P. Mather ${ }^{1}$, R. Atkinson ${ }^{2}$, J.S. Neto ${ }^{3}$, M.F. Q Vieira ${ }^{3}$, P. Lazaridis ${ }^{1}$ and I.A. Glover ${ }^{1}$ \\ ${ }^{1}$ Department of Engineering \& Technology, University of Huddersfield, Huddersfield HD1 3DH, UK, \\ http://www.hud.ac.uk/ce/ \\ ${ }^{2}$ Department of Electrical Engineering, University Federal de Campina Grande, Campina Grande, Brazil \\ ${ }^{3}$ Deptartment of Electronic and Electrical Engineering, University of Strathclyde, Glasgow G1 1XW, UK
}

Progress on the development of an insulation defect detection and location system using a partial discharge (PD) wireless sensor network (WSN) will be presented. Such a PD WSN based on intensity-

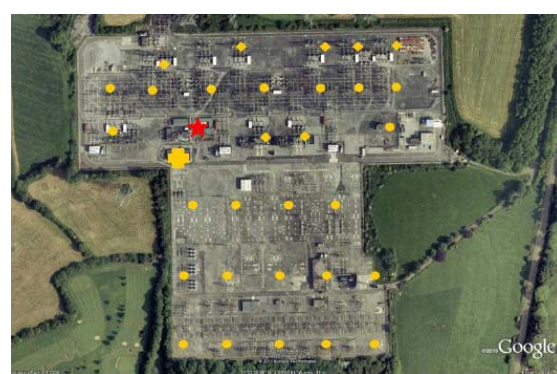

Fig. 1 Hypothetical PD WSN Example only measurements has cost and scalability advantages over existing detection and location technologies based on timedifference-of-arrival measurements such as described in (I. E. Portugues, P. J. Moore, I. A. Glover, IEEE Trans. on Power Delivery, 1, 2009, pp. 20-29). Figure 1 shows a hypothetical deployment of the PD WSN in an electricity substation. The (red) pentagram denotes a PD source, yellow circles and triangles denote sensor nodes, and the yellow St George's cross denotes the data collection/processing node. Each node of the WSN is a broadband radiometer with a measurement band of 50-800 MHz, Figure 2. Three measurement sub-bands allow the radiometer to distinguish different forms of PD; in particular internal PD and corona discharge. WirelessHart has been selected as the network communications technology since this offers improved reliability over other standards (e.g. Zigbee) in harsh industrial environments.

In the substation environment the path-loss index is both unknown and generally different for the path
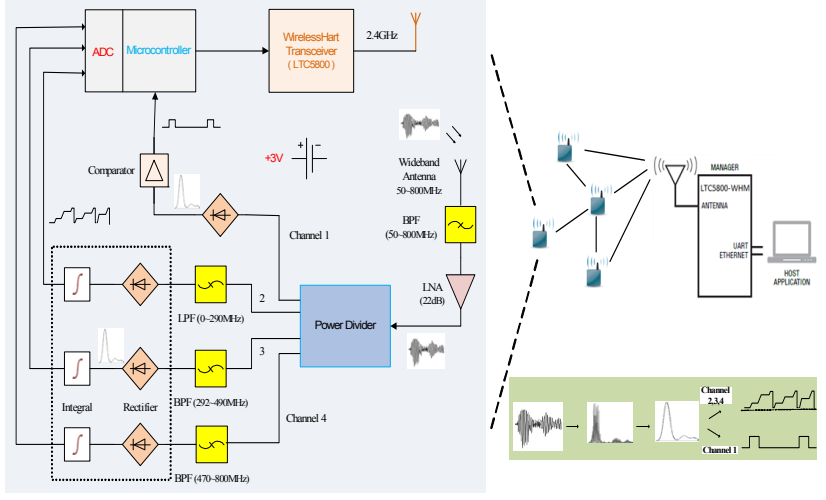

Fig. 2 Multichannel PD Sensor and WirelessHart Node from the PD source to each sensor, so range cannot be estimated from a single power measurement. A simulated PD signal will therefore be periodically radiated from each node to calibrate path-loss index from that node to all other nodes. The authors' (currently) favoured PD location method is based on a variation of path-loss inversion as follows: (i) assume a plausible path-loss index (e.g. $n=2$ ), (ii) calculate the ratio of received power at any two nodes implying a locus of possible source locations, (iii) use intersecting loci from all measurement node pairs to establish an initial

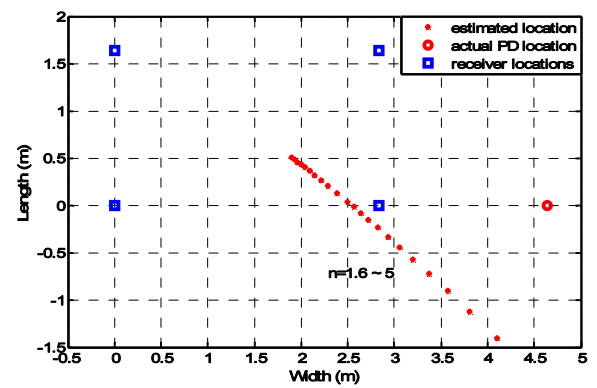

Fig. 3 Experimental site map and results estimate of source location, (iv) use the initial source location to refine the estimate of $n$ appropriate to each sensing node, (v) use pairs of nodes corresponding to paths with (nearly) equal $n$ to calculate an improved estimate of source location, (vi) iterate as necessary to converge on a final location estimate.

Proof-of-principle measurements using an artificial PD source and a sensor node in Fig. 2 in a laboratory environment are encouraging, Figure 3. These proof-of-principle results implement only steps (i) through (iii) of the algorithm described above. Steps (iv) through (vi) of the algorithm will result in a final location accuracy at least as good as selecting the optimum path-loss index in Figure 3 and probably better than this. The error between estimated and actual PD source location selecting the optimum path-loss index is $1.4 \mathrm{~m}$. 